Production, Manufacturing and Logistics

\title{
Non-cooperative joint replenishment under asymmetric information
}

\author{
Evren Körpeoğlu ${ }^{a}$, Alper Şen ${ }^{\mathrm{b}, *}$, Kemal Güler ${ }^{\mathrm{a}}$ \\ ${ }^{a}$ Hewlett-Packard Laboratories, MS 1140, Palo Alto, CA 94304, USA \\ ${ }^{\mathrm{b}}$ Department of Industrial Engineering, Bilkent University, Bilkent, Ankara 06800, Turkey
}

\section{A R T I C L E I N F O}

\section{Article history:}

Received 15 September 2012

Accepted 2 January 2013

Available online 11 January 2013

\section{Keywords:}

Inventory

Joint replenishment

Economic Order Quantity model

Non-cooperative game theory

Information asymmetry

\begin{abstract}
A B S T R A C T
We consider jointly replenishing $n$ ex-ante identical firms that operate under an EOQ like setting using a non-cooperative game under asymmetric information. In this game, each firm, upon being privately informed about its demand rate (or inventory cost rate), submits a private contribution to an intermediary that specifies how much it is willing to pay for its replenishment per unit of time and the intermediary determines the maximum feasible frequency for the joint orders that would finance the fixed replenishment cost. We show that a Bayesian Nash equilibrium exists and characterize the equilibrium in this game. We also show that the contributions are monotone increasing in each firm's type. We finally conduct a numerical study to compare the equilibrium to solutions obtained under independent and cooperative ordering, and under full information. The results show that while information asymmetry eliminates free-riding in the contributions game, the resulting aggregate contributions are not as high as under full information, leading to higher aggregate costs.
\end{abstract}

(c) 2013 Elsevier B.V. All rights reserved.

\section{Introduction}

A fundamental trade-off in operations is between cycle stocks and setup costs associated with production, transportation or procurement. Mathematical models examining this trade-off have been developed since 1913 starting with the classical Economic Order Quantity (EOQ) model (Harris, 1913). In the EOQ model, a firm faces a constant and deterministic demand rate, pays a fixed setup cost for each replenishment order and incurs a holding cost for each unit of inventory it keeps per unit of time. The problem is to find the order quantity that the firm should use in each replenishment so that its setup costs and inventory holding costs are minimized. Since then, there has been a vast amount of literature on lot sizing that relaxes certain restrictive assumptions of the EOQ model. The interested reader is referred to Jans and Degraeve (2008) for a recent review of research in this area.

A major cost saving opportunity in this setting is joint replenishment, i.e., consolidating orders for different items (or locations). By carefully coordinating the replenishment of multiple items, one can exploit the economies of scale of ordering jointly and reduce setup costs, cycle inventories or both. Finding a joint replenishment policy to minimize aggregate costs is known as the joint replenishment problem in the literature. There is also large body of research in this area: see Khouja and Goyal (2008) for a recent review.

\footnotetext{
* Corresponding author. Tel.: +90 312 2901539; fax: +90 3122664054 .

E-mail address: alpersen@bilkent.edu.tr (A. Şen).
}

Although joint replenishment may be a significant means to reduce costs, when it involves a group of items or locations that are not controlled centrally, it is not always apparent how to split these savings among the parties fairly. A fair allocation is necessary to induce different decentralized entities to engage in cooperation. Recently, cooperative game theory models are developed to investigate whether a fair allocation of total savings (or total costs) is possible and if so, how. In the first of these models, Meca et al. (2004) show that it is possible to coordinate the system (obtain minimum total cost) when the players only share their order frequencies prior to joint replenishment. They propose an allocation mechanism which distributes the total setup cost among the jointly replenished locations in proportion to the square of their order frequencies and show that this allocation is in the core of the game, i.e., the firms cannot decrease their costs further by defecting from the grand coalition of firms. In Meca et al. (2004), there are only major setup costs, i.e., setup costs are independent of which items are included in the order. When there are also minor setup costs associated with each item, it is not always optimal to order every item with every replenishment. In fact, the structure of the policy that minimizes the total costs is not known. For this problem, Hartman and Dror (2007) show that the game with a specific group of items has a core, whenever these items need to be ordered together on the same schedule to minimize total costs. For the same problem, Dror et al. (2012) investigate how sensitive the stability of coordinated ordering (or the existence of a core) is to the changes in the cost parameters and conduct a computational study to show that a core allocation can be obtained without excessive computation. In particular, the Lauderback allocation 
(each firm's allocation is in proportion to a weighted average of its stand-alone cost and marginal cost) is in the core for $98.8 \%$ of the games they study. Elomri et al. (2012) consider the problem of coalition formation when the joint replenishment game is not superadditive and propose an exact fractional programming based solution to find the efficient coalitions. Anily and Haviv (2007) limit their attention to the near optimal power-of-two policies for this problem, and show the existence and example of a core allocation of total costs. These results are extended to the case of more general setup cost structures in Zhang (2009). In a related paper, Viswanathan and Piplani (2001) study a problem of a vendor which coordinates the replenishment of multiple retailers by requiring them to order at multiples of a common replenishment period and offering a price discount to entice the retailers to accept this strategy. Minner (2007) uses bargaining models to study the collaboration between two firms that have a common interest to jointly replenish material requirements. Van den Heuvel et al. (2007) consider a problem in which multiple retailers have to satisfy their periodic, non-stationary and known demand over a finite horizon. The authors study the cooperative game that arises from cost savings obtained by jointly replenishing the retailers. Recent reviews of cooperative games for joint replenishment and other inventory problems can be seen in Nagarajan and Sošić (2008) and Fiestras-Janeiro et al. (2011).

While cooperative analysis of joint replenishment problems received a reasonable amount of attention in the literature, there is limited research that use non-cooperative game theory to study such problems. In fact, Bauso et al. (2008), Meca et al. (2003) and Körpeoğlu et al. (2012) are the only three papers that adopt a non-cooperative approach. Bauso et al. (2008) study a finite horizon, periodic problem in which multiple firms need to determine their order quantities in each period to satisfy their demand. The replenishment order cost is shared among multiple firms that order in the same period. It is shown that this game admits a set of pure-strategy Nash equilibria, one of which is Pareto optimal. The authors propose a consensus protocol with which firms converge to one of the Nash equilibria, but not necessarily a Pareto optimal one.

Meca et al. (2003) study a non-cooperative reporting game where stand-alone order frequencies of the firms are common knowledge but not verifiable. Each firm reports an order frequency (that may be different from its true order frequency) and the joint order frequency is determined to minimize the total joint costs based on these reports. Each firm incurs holding cost individually and pays a share of the joint replenishment cost in proportion to the squares of reported order frequencies. It is shown that while this rule leads to core allocations under cooperative formulations, it results in significant misreporting and inefficiency in a noncooperative framework.

Körpeoğlu et al. (2012) use a more direct approach. They consider $n$ firms with arbitrary demand and inventory holding cost rates. These are assumed to be common knowledge. There is a fixed replenishment cost which can be incurred individually by each firm or incurred jointly among firms that would participate in joint replenishment. Each firm decides whether to participate in joint replenishment or to replenish independently and each participating firm reports the level of his contribution to an intermediary. The intermediary then selects the smallest joint cycle time that can be financed with these contributions. Körpeoğlu et al. (2012) find that in any equilibrium of the game, the firms with the lowest stand-alone cycle time share the replenishment cost and the others pay only the minimum contribution.

An important assumption used in all papers that study noncooperative joint replenishment games is that all relevant information is common knowledge. However, as in many other contexts, information asymmetries exist in many practical supply chain settings due to lack of communication or incentives for hiding information. It is often crucial to model these asymmetries in order to better understand the strategic behavior among competing firms or design mechanisms that would lead to more favorable outcomes for them. Recently, we have seen studies in supply chain management literature that model information asymmetries. Important examples include Corbett (2001) and Burnetas et al. (2007).

Previous research on the joint replenishment problem highlights the importance of modeling private information in this particular setting. As mentioned above, in the equilibrium found in Körpeoğlu et al. (2012), firms with the lowest stand-alone cycle time pay for all of the replenishment cost except the minimum contributions that will be paid by the rest of the firms. Thus, if a firm knows that there is another firm with a lower stand-alone cycle time (or higher adjusted demand rate which is equal to inventory holding cost rate multiplied by the demand rate), it tends to ride free and pays the minimum contribution. In an asymmetric information game, however, we should get larger contributions from the firms with higher stand-alone cycle times, since the other firms' information is not available to them. Consequently, an important question is whether removing the possibility of free-riding through introducing information asymmetry would lead to a more efficient mechanism in terms of aggregate costs incurred by all firms.

In this paper, we consider the problem of jointly replenishing $n$ firms that operate under an EOQ like setting using a non-cooperative game in which firms are privately informed about their parameters (demand rates and/or inventory holding cost rates). The game we model is an asymmetric information counterpart of the game suggested in Körpeoğlu et al. (2012). Each firm, upon being privately informed about its parameters, submits a private contribution to an intermediary that specifies how much it is willing to contribute to replenishment costs per unit of time. The intermediary then determines the frequency for the joint orders that would finance the setup cost. Since each firm's inventory holding cost depends on the joint frequency which in turn depends on contributions from all firms, this is non-cooperative game in which each firm's strategy is its contribution. Our solution concept for this game is Bayesian Nash equilibrium. A Bayesian Nash equilibrium is a Nash equilibrium where each player, given its type (parameter), selects a best response against the average best responses of the competing players. We prove that a pure-strategy Bayesian Nash equilibrium exists and show that the equilibria can be characterized by a system of integral equations. We also conduct a numerical study to analyze the impact of competition and information asymmetry on equilibrium behavior and outcomes by comparing the equilibrium we obtain to centralized and independent ordering and to a non-cooperative game under full information. The results show that joint replenishment continues to lead to significant improvements over independent ordering, despite the fact that firms compete under information asymmetry. The results also show that while information asymmetry eliminates free-riding in the game, the full information game leads to more efficient outcomes.

The rest of this paper is organized as follows. In Section 2, we provide our model and results for the asymmetric information game. In Section 3, we provide the results of our numerical study and the managerial insights derived from this study. Section 4 concludes the paper along with avenues for future research.

\section{Model}

We consider a stylized EOQ environment with a set of firms $N=\{1, \ldots, n\}(|N|=n)$. Each firm $j$ is facing a constant deterministic demand with rate $\beta_{j}$ per unit of time. Inventory holding cost rate 
is $\gamma_{j}$ per unit of time. Major ordering cost is fixed at $\kappa$ per order regardless of order size and we assume minor ordering costs are zero. We define $\alpha_{j}=\gamma_{j} \beta_{j}$, which will be convenient in all the settings that we consider below. We will refer to $\alpha_{j}$ as adjusted demand rate for firm $j$. We assume that each firm's adjusted demand rate is its private information. We also assume that $\alpha_{j}$ s are independent draws from a common continuous prior distribution $F$ with support $A=[\underline{\alpha}, \bar{\alpha}]$ with $0<\underline{\alpha}<\bar{\alpha}<+\infty$. Note that this captures having uncertainty on demand rate or inventory holding cost rate (given that the other is same across firms) or on both demand rate and inventory holding cost rate.

In the game we propose, first, each firm learns its adjusted demand rate (type). Then, firms submit their private contributions that specify their payment rate for the replenishment service. Based on these contributions, the intermediary then determines the minimum cycle length of the joint replenishment such that would finance the fixed cost $\kappa$. Finally, firms incur their costs according to this cycle length. Since firms do not reveal their type during the game, we have an asymmetric information game in which each firm's strategy is its contribution, which is a function of its adjusted demand rate.

Let $r_{j}: A \rightarrow \Theta$ be the contribution function where $\Theta=[0, \bar{r}]$ and $r_{j}\left(\alpha_{j}\right)$ is the contribution that firm $j$ makes if its type is $\alpha_{j}$. We assume an upper bound $\bar{r}=\sqrt{2 \kappa \bar{\alpha}}$ on the action space since a contribution higher than this value leads to a total cost higher than the stand-alone total cost regardless of the adjusted demand rate realizations. Moreover, we exclude negative contributions. Then, for a given $\alpha=\left(\alpha_{1}, \ldots, \alpha_{n}\right)$ the intermediary will set the cycle length to

$t(\boldsymbol{\alpha})=\frac{\kappa}{\sum_{k \in N} r_{k}\left(\alpha_{k}\right)}$.

Consider a firm $j$ with type $\alpha_{j}$. Denote $\mathbf{r}_{-j}\left(\boldsymbol{\alpha}_{-j}\right)$ as the vector of contributions of the firms except that of firm $j$ under realization $\boldsymbol{\alpha}_{-\mathbf{j}}=\left(\alpha_{1}, \ldots, \alpha_{j-1}, \alpha_{j}, \ldots, \alpha_{n}\right)$. The payoff for firm $j$ under this realization can be written as

$\phi_{j}\left(r_{j}, \boldsymbol{r}_{-\mathbf{j}}, \alpha_{j}, \boldsymbol{\alpha}_{-\mathbf{j}}\right)=\frac{1}{2} \alpha_{j} t\left(\alpha_{j}, \boldsymbol{\alpha}_{-\mathbf{j}}\right)+r_{j}$,

and the expected payoff for this firm is

$$
\begin{aligned}
\Phi_{j}\left(r_{j}, \boldsymbol{r}_{-\mathbf{j}}, \alpha_{j}\right) & =\int_{A^{n-1}} \phi\left(r_{j}, \boldsymbol{r}_{-\mathbf{j}}, \alpha_{j}, \boldsymbol{\alpha}_{-\mathbf{j}}\right) f^{n-1}\left(\boldsymbol{\alpha}_{-\mathbf{j}}\right) \mathrm{d} \boldsymbol{\alpha}_{-\mathbf{j}} \\
& =\frac{1}{2} \kappa \alpha_{j} \int_{A^{n-1}} \frac{1}{r_{j}+\sum_{k \in N, k \neq j} r_{k}\left(\alpha_{k}\right)} f^{n-1}\left(\boldsymbol{\alpha}_{-\mathbf{j}}\right) \mathrm{d} \boldsymbol{\alpha}_{-\mathbf{j}}+r_{j}
\end{aligned}
$$

where $A^{n-1}$ is the $(n-1)$ st Cartesian power of the interval $A$ and $f^{n-1}\left(\boldsymbol{\alpha}_{-\mathbf{j}}\right)=\prod_{k \in N, k \neq j} f\left(\alpha_{k}\right)$.

Firm $j$ 's best response as a function of its type $\alpha_{j}$ and other firms' contributions $\boldsymbol{r}_{-\mathbf{j}}$ is denoted by $\rho$ and is given by

$\rho\left(\boldsymbol{r}_{-\mathbf{j}}, \alpha_{j}\right)=\arg \min _{r_{j}} \Phi_{j}\left(r_{j}, \boldsymbol{r}_{-\mathbf{j}}, \alpha_{j}\right)$ subject to $r_{j} \geqslant 0$.

The first and second derivative of the payoff function (3) are given as follows

$\frac{\partial \Phi_{j}\left(r_{j}, \boldsymbol{r}_{-\mathbf{j}}, \alpha_{j}\right)}{\partial r_{j}}=-\frac{1}{2} \kappa \alpha_{j} \int_{A^{n-1}} \frac{1}{\left(r_{j}+\sum_{k \in N, k \neq j} r_{k}\left(\alpha_{k}\right)\right)^{2}} f^{n-1}\left(\boldsymbol{\alpha}_{-\mathbf{j}}\right) \mathrm{d} \boldsymbol{\alpha}_{-\mathbf{j}}+1$,

$\frac{\partial^{2} \Phi_{j}\left(r_{j}, \boldsymbol{r}_{-\mathbf{j}}, \boldsymbol{\alpha}_{j}\right)}{\partial r_{j}^{2}}=\kappa \alpha_{j} \int_{A^{n-1}} \frac{1}{\left(r_{j}+\sum_{k \in N, k \neq j} r_{k}\left(\alpha_{k}\right)\right)^{3}} f^{n-1}\left(\boldsymbol{\alpha}_{-\mathbf{j}}\right) \mathrm{d} \boldsymbol{\alpha}_{-\mathbf{j}}$

Since contributions are non-negative, the integrand in (6) is a non-negative function leading to the convexity of the payoff function in $r_{j}$. Therefore, the problem (4) is a convex optimization problem with a single constraint. The problem has a corner solution and firm $j$ 's best response is to contribute zero if the first derivative of the objective function given in (5) is non-negative at $r_{j}=0$ and an interior solution (characterized by the first order condition), otherwise. Therefore, the best response function can be expressed as follows:

$\rho_{j}\left(\boldsymbol{r}_{-\mathbf{j}}, \alpha_{j}\right)= \begin{cases}0, & \text { if } \int_{A^{n-1}} \frac{1}{\left(\sum_{k \in N, k \neq j} r_{k}\left(\alpha_{k}\right)\right)^{2}} f^{n-1}\left(\boldsymbol{\alpha}_{-\mathbf{j}}\right) \mathrm{d} \boldsymbol{\alpha}_{-\mathbf{j}} \leqslant \frac{2}{k \alpha_{j}}, \\ \hat{\rho}\left(\boldsymbol{r}_{-\mathbf{j}}, \alpha_{j}\right), & \text { otherwise, }\end{cases}$

where $\hat{\rho}\left(\boldsymbol{r}_{-\mathbf{j}}, \alpha_{j}\right)$ is the solution to

$\int_{A^{n-1}} \frac{1}{\left(\hat{\rho}\left(\boldsymbol{r}_{-\mathbf{j}}, \alpha_{j}\right)+\sum_{k \in N, k \neq j} r_{k}\left(\alpha_{k}\right)\right)^{2}} f^{n-1}\left(\boldsymbol{\alpha}_{-\mathbf{j}}\right) \mathrm{d} \boldsymbol{\alpha}_{-\mathbf{j}}=\frac{2}{\kappa \alpha_{j}}$.

The characterization in (7) states that a firm $j$ with type $\alpha_{j}$ will choose to ride free $\left(r_{j}=0\right)$ if the second moment of the joint cycle time among the other $n-1$ firms is smaller than the square of stand-alone cycle time for firm $j$. Alternatively, (7) states that given the contribution strategies of others, there will be a threshold value for firm $j$ 's adjusted demand rate below which the firm will contribute zero. This threshold is given by

$\hat{\alpha}\left(\mathbf{r}_{-\mathbf{j}}\right)=\frac{2}{\kappa \int_{A^{n-1}} \frac{1}{\left(\sum_{k \in N, k \neq j} r_{k}\left(\alpha_{k}\right)\right)^{2}} f^{n-1}\left(\boldsymbol{\alpha}_{-\mathbf{j}}\right) \mathrm{d} \boldsymbol{\alpha}_{-\mathbf{j}}}$.

Convexity of the objective function also ensures that each firm has a unique best response to other firms' contributions given its type.

We next show that each firm's best response function is increasing in its type (all proofs are provided in Appendix).

Lemma 1. Each firm j's best response to other firms' contributions is increasing in its type $\alpha_{j}$.

A pure-strategy Bayesian Nash equilibrium is a set of functions $\boldsymbol{r}^{*}=\left(r_{1}^{*}, r_{2}^{*}, \ldots, r_{n}^{*}\right)$ such that $\rho_{j}\left(\boldsymbol{r}_{-\mathbf{j}}^{*}, \alpha_{j}\right)=r_{j}^{*}\left(\alpha_{j}\right)$ for all $\alpha_{j}$ and for all $j \in N$. We establish the existence of a pure-strategy Bayesian Nash equilibrium in the next theorem.

Theorem 1. A pure-strategy Bayesian Nash equilibrium exists for the joint replenishment game under asymmetric information.

In order to characterize the Bayesian Nash equilibria, we need the following lemma which states that there is at least one firm which contributes a positive amount regardless of its type.

Lemma 2. In any equilibrium, there exists at least one firm $j$ such that $r_{j}^{*}\left(\alpha_{j}\right)>0, \quad$ forall $\alpha_{j} \in A$.

Using Lemma 2, we can provide a simple characterization of the Bayesian Nash equilibria in the following theorem.

Theorem 2. Any collection of functions $\left(r_{1}^{*}\left(\alpha_{1}\right), r_{2}^{*}\left(\alpha_{2}\right), \ldots, r_{n}^{*}\left(\alpha_{n}\right)\right)$ that satisfy (9) is a Bayesian Nash equilibrium.

$$
\begin{aligned}
& \int_{A^{n-1}} \frac{1}{\left(r_{1}^{*}\left(\alpha_{1}\right)+r_{2}^{*}\left(\alpha_{2}\right)+\ldots+r_{n}^{*}\left(\alpha_{n}\right)\right)^{2}} f^{n-1}\left(\boldsymbol{\alpha}_{-\mathbf{j}}\right) \mathrm{d} \boldsymbol{\alpha}_{-\mathbf{j}} \\
& \quad=\frac{2}{\kappa \alpha_{j}}, \quad \text { for all } j \in N .
\end{aligned}
$$

Theorem 2 states that finding an equilibrium requires solving $n$ integral equations simultaneously. Obtaining a closed form solution for the system (9) is not possible. However, we can provide the following properties for the equilibrium contribution functions.

First, if we multiply both sides of (9) by $\kappa^{2} f\left(\alpha_{j}\right)$ and integrate both sides over the interval $A$, we get

$\mathbb{E}\left[\left(\frac{\kappa}{\left(r_{1}^{*}\left(\alpha_{1}\right)+r_{2}^{*}\left(\alpha_{2}\right)+\cdots+r_{n}^{*}\left(\alpha_{n}\right)\right)}\right)^{2}\right]=\mathbb{E}\left[\frac{2 \kappa}{\alpha_{j}}\right]$. 
Using (1), one can see that the left hand side is the second moment of the equilibrium cycle length. The right hand side, on the other hand is the second moment of the stand-alone cycle length. This shows that second moment of cycle length is "invariant" - it is preserved when one moves from stand-alone (independent) replenishment to non-cooperative joint replenishment.

In addition, using the monotonicity of the best-response functions provided in Lemma 1, we can also say the following regarding the equilibrium contribution functions.

Corollary 1. In all equilibria, the contribution function for each firm is monotone increasing in its type.

The characterization in (9) of Theorem 2 allows multiple equilibria with different contribution functions for each player. However, if we restrict ourselves to symmetric equilibria, we have the following result.

Corollary 2. The symmetric Bayesian Nash equilibrium satisfies the following

$\int_{A^{n-1}} \frac{1}{\left(r^{*}\left(\alpha_{j}\right)+\sum_{k \in N, k \neq j} r^{*}\left(\alpha_{k}\right)\right)^{2}} f^{n-1}\left(\boldsymbol{\alpha}_{-\mathbf{j}}\right) \mathrm{d} \boldsymbol{\alpha}_{-\mathbf{j}}=\frac{2}{\kappa \alpha_{j}}$ for all $\alpha_{j}$.

Now consider the symmetric equilibrium $r^{*}$. For a given realization $\boldsymbol{\alpha}=\left(\alpha_{1}, \ldots, \alpha_{n}\right)$, the cycle length that is set by the intermediary is given as

$T^{a}(\boldsymbol{\alpha})=\frac{\kappa}{\sum_{k \in N} r^{*}\left(\alpha_{k}\right)}$.

This leads to an aggregate total cost expression as follows

$C^{a}(\boldsymbol{\alpha})=\frac{1}{2} \frac{\kappa \sum_{k \in N} \alpha_{k}}{\sum_{k \in N} r^{*}\left(\alpha_{k}\right)}+\sum_{k \in N} r^{*}\left(\alpha_{k}\right)$.

Therefore expected replenishment cost, and expected aggregate total cost rate can be written as

$$
\begin{aligned}
& \mathbb{E}\left[R^{a}\right]=n \int_{A} r^{*}(\alpha) f(\alpha) \mathrm{d} \alpha, \\
& \mathbb{E}\left[C^{a}\right]=\frac{1}{2} \kappa \int_{A^{n}} \frac{\sum_{k \in N} \alpha_{k}}{\sum_{k \in N} r^{*}\left(\alpha_{k}\right)} f^{n}(\boldsymbol{\alpha}) \mathrm{d} \boldsymbol{\alpha}+\mathbb{E}\left[R^{a}\right] .
\end{aligned}
$$

\subsection{Possible extensions}

It is important to note the implications of relaxing certain assumptions on our model and results. First, we assume that the adjusted demand rates are independent draws from the same distribution. In general, each player's adjusted demand rate may be drawn from a different distribution. Furthermore, each player's adjusted demand rate estimate for another player may be different. Let, $F_{k}^{j}$ be the distribution of player $j$ 's estimate of player $k$ with a support $A_{k}^{j}$. One can verify that the existence result provided in Theorem 1 still holds for this case. One can also show that the equilibrium can now be characterized as

$$
\int_{A^{-\mathbf{j}}} \frac{1}{\left(r_{1}^{*}\left(\alpha_{1}\right)+r_{2}^{*}\left(\alpha_{2}\right)+\cdots+r_{n}^{*}\left(\alpha_{n}\right)\right)^{2}} f^{-\mathbf{j}}\left(\boldsymbol{\alpha}_{-\mathbf{j}}\right) \mathrm{d} \boldsymbol{\alpha}_{-\mathbf{j}}=\frac{2}{\kappa \alpha_{j}},
$$

for all $j \in N$,

where $A^{-\mathbf{j}}=\prod_{k \in N, k \neq j} A_{k}^{j}, f_{k}^{j}=\mathrm{d} F_{k}^{j}$ and $f^{-\mathbf{j}}\left(\boldsymbol{\alpha}_{-\mathbf{j}}\right)=\prod_{k \in N, k \neq j} f_{k}^{j}\left(\alpha_{k}\right)$. While this extension does not create any theoretical difficulties, computing the equilibrium will be challenging as one needs to solve a system of $n$ integral equations. In our numerical analysis in Section 3, we assume a common distribution which allows us to focus on the symmetric equilibrium that requires solving only one integral equation.

Second, we assume that the setup cost $\kappa$ is known with certainty by all parties. Having an uncertainty or information asymmetry on $\kappa$ will require a firm to take this into account when minimizing its expected payoff in (3). We also assume that each firm knows its own adjusted demand rate prior to submitting its contribution. In a more general model, a firm may have uncertainty regarding its own demand rate as well (although the uncertainty it faces will be obviously smaller than what the other firms are exposed to regarding its adjusted demand rate). Again this uncertainty has to be considered in the objective function of the firm in (3). While our existence result should hold in these cases, we leave these extensions to a future study.

Finally, we assume that working with an intermediary does not lead to any coordination costs such as cost of exchanging information and incorporating that information to the joint decisions, as well as costs incurred by the firm due to delays (Clemons et al., 1993). In the absence of these costs and minor setup costs, firms will prefer participating to joint replenishment over independent ordering, and therefore we do not model opting out from joint replenishment in this study (please see Körpeoğlu et al. (2012) for such a game). Once the contributions game is played, the maximum coordination cost that each player is willing to pay will be the difference between a firm's cost in joint replenishment equilibrium and its stand-alone cost. In other cases, we need a formal game in which these costs are represented as a part of each player's payoff (especially when the coordination cost is a function of the number of participants in joint replenishment). We also leave this important extension to a future study.

\subsection{Benchmark models}

We now briefly review the three benchmark models used for comparison: independent (decentralized) replenishment, joint (centralized) replenishment and competitive replenishment under full information.

INDEPENDENT (DECENTRALIZED) REPLENISHMENT

When the replenishment of the items is controlled by firms operating independently, firm $j$ 's total cost rate $\left(C_{j}\right)$ is the sum of its replenishment cost rate $\left(R_{j}\right)$ and holding cost rate $\left(H_{j}\right)$ and can be written as a function of the cycle time $t$ as follows

$C_{j}(t)=R_{j}(t)+H_{j}(t)=\frac{\kappa}{t}+\frac{t}{2} \alpha_{j}$.

We assume that each firm learns its adjusted demand rate (type) prior to determining its cycle length. Therefore, the function in (11) can be minimized with the realized value of $\alpha_{j}$. This leads to firm $j$ 's optimal cycle time $T_{j}^{d}=\sqrt{2 \kappa / \alpha_{j}}$. Resulting replenishment cost rate is $R_{j}^{d}=\sqrt{\kappa \alpha_{j} / 2}$. Holding cost rate for firm $j$ is also $H_{j}^{d}=\sqrt{\kappa \alpha_{j} / 2}$. Thus firm $j$ 's total cost per unit of time is $C_{j}^{d}=\sqrt{2 \kappa \alpha_{j}}$. The aggregate total cost rates for $n$ firms under independent replenishment are $C^{d}=\sum_{k \in N} \sqrt{2 \kappa \alpha_{k}}$, and $R^{d}=H^{d}=$ $\sum_{k \in N} \sqrt{\kappa \alpha_{k} / 2}$.

JoINT (CENTRALIZED) REPLENISHMENT

In joint replenishment, replenishment decisions are taken centrally to minimize the aggregate total cost. When there are no minor setup costs (setup costs specific to each firm), all firms will be replenished in each cycle leading to a common cycle time (see Meca et al. (2004) for a proof). The aggregate cost for $n$ firms as a function of the common cycle time $t$ can be written as

$C^{c}(t)=R^{c}(t)+H^{c}(t)=\frac{\kappa}{t}+\frac{t}{2} \sum_{k \in N} \alpha_{k}$.

Once again, we assume that the values of $\alpha_{k}$ are known before the joint cycle time decision is taken. Therefore, the optimal cycle time 
can be found by minimizing (12) as $T^{c}=\sqrt{2 \kappa / \sum_{k \in N} \alpha_{k}}$. Then, the optimal cost rates are $C^{c}=\sqrt{2 \kappa \sum_{k \in N} \alpha_{k}}$, and $R^{c}=H^{c}=C^{c} / 2$.

COMPETITIVE RePlenishment under Full INFORMATION

Under full information, firms reveal their adjusted demand rates (types) prior to submitting their private contributions. Therefore, each firm uses its type information, as well as others' when deciding its contribution. The resulting game is simply the onestage game described in Körpeoğlu et al. (2012) with minimum contribution $\delta=0$. Let $\alpha_{1}, \alpha_{2}, \ldots, \alpha_{n}$ be the types of the firms $1, \ldots, n$. Let $(n)$ be the firm with the highest adjusted demand rate, i.e., $\alpha_{(n)}=\max _{j \in N} \alpha_{j}$. Also let $L=\left\{j \in N \mid \alpha_{j}=\alpha_{(n)}\right\}$. Körpeoğlu et al. (2012) show that the equilibrium contributions satisfy the following properties:

$r_{k}^{*}=0, \quad \forall k \in N \backslash L$, and $\sum_{k \in L} r_{k}^{*}=\sqrt{\kappa \alpha_{(n)} / 2}$.

That is, firms whose adjusted demand rates are lower than the highest adjusted demand rate do not contribute for the joint replenishment and ride free. Firms with the highest adjusted demand rate, on the other hand, contribute a total of $\sqrt{\kappa \alpha_{(n)} / 2}$. The equilibrium is unique if there is only one firm with the highest adjusted demand rate ( $L$ is a singleton), otherwise there are multiple equilibria. However, in all equilibria, aggregate contributions, aggregate costs and the joint cycle time are unique. Joint cycle time is $T^{f}=\sqrt{2 \kappa / \alpha_{(n)}}$ Aggregate replenishment cost is $R^{f}=\sum_{k \in N} r_{k}^{*}=\sqrt{\kappa \alpha_{(n)} / 2}$ and aggregate total cost is $C^{f}=\sqrt{\kappa / 2 \alpha_{(n)}}\left(\alpha_{(n)}+\sum_{k \in N} \alpha_{k}\right)$.

The above characterization states that the equilibrium is determined only by the largest adjusted demand rate. Since adjusted demand rates are independent and identically distributed random variables, this corresponds to the largest order statistic. Using this fact, we can obtain the following expressions for the expected aggregate replenishment cost, and expected aggregate total cost:

$\mathbb{E}\left[R^{f}\right]=n \int_{A} \sqrt{\frac{\kappa \alpha}{2}} f(\alpha)[F(\alpha)]^{n-1} \mathrm{~d} \alpha$,

$\mathbb{E}\left[C^{f}\right]=n ! \int_{\underline{\alpha}}^{\bar{\alpha}} \int_{\underline{\alpha}}^{\alpha_{n}} \cdots \int_{\underline{\alpha}}^{\alpha_{2}} \sum_{k \in N} \alpha_{k} \sqrt{\kappa / 2 \alpha_{n}} f^{n}(\boldsymbol{\alpha}) \mathrm{d} \boldsymbol{\alpha}+\mathbb{E}\left[R^{f}\right]$.

The expression in (14) is due to the fact that largest order statistic $\alpha_{(n)}$ has a probability density function equal to $n f(\alpha)[F(\alpha)]^{n-1}$. The expression in (15) is due to the fact that $\alpha_{(1)}, \alpha_{(2)}, \ldots, \alpha_{(n)}$ have a joint density $n ! f^{n}(\boldsymbol{\alpha})$.

\section{Numerical study}

We conduct a computational study to understand the impact of competition and information asymmetry on equilibrium behavior and total costs. In doing this, we compare the asymmetric information game to centralized replenishment, independent replenishment and competitive replenishment under full information. To set up the experiment, the only two variables we need are the replenishment fixed cost $\kappa$ and the probability distribution of adjusted demand rates $\alpha_{j}$. The choice of the fixed cost $\kappa$ is immaterial as it appears only as a multiple in cost expressions and cancels out in comparisons. We use a modified PERT distribution (Vose, 2008) to model the uncertainty in adjusted demand rates. The modified PERT distribution is a 4-parameter distribution and is frequently used to model expert data. Expert opinion is used to specify the minimum $(a)$, maximum $(b)$, most-likely $(m)$ values and a fourth parameter $(\lambda)$ controls the shape. The modified PERT distribution is given as follows:

$f(x)=\frac{(x-a)^{\eta_{1}-1}(b-x)^{\eta_{2}-1}}{B\left(\eta_{1}, \eta_{2}\right)(b-a)^{\eta_{1}+\eta_{2}-1}}$, where $B\left(\eta_{1}, \eta_{2}\right)$ is the beta function, and

$\eta_{1}=1+\lambda\left(\frac{m-a}{b-a}\right), \quad \eta_{2}=1+\lambda\left(\frac{b-m}{b-a}\right)$.

The mean $(\mu)$ and variance $\left(\sigma^{2}\right)$ are

$\mu=\frac{a+\lambda m+b}{\lambda+2}, \quad \sigma^{2}=\frac{(\mu-a)(b-\mu)}{\lambda+3}$.

We pick the modified PERT distribution since it is defined over a finite interval and we can easily control the skewness and mode with two dedicated parameters. In order to demonstrate the flexibility of the modified PERT distribution, we plot the density for various values of $m$ and $\lambda$ for $(a, b)=(1,5)$ in Fig. 1 . Observe that when $\lambda=0$, the distribution reduces to uniform distribution.

We start our computational study with understanding the equilibrium behavior in the non-cooperative asymmetric information game. For this purpose, we assume that the adjusted demand rates are independently and identically distributed with modified PERT distribution with parameters $a=1, b=5$ and various values of $\lambda$ and $m$. The fixed cost $\kappa$ is equal to 10 . We consider only the symmetric equilibrium. In order to compute the equilibrium given in Eq. (10) numerically, we discretize the distribution of $\alpha$ at forty equally spaced points in the interval $(a, b)$.

Fig. 2 shows the contribution of a single firm as a function of its adjusted demand rate when there are $1,2,3$, or 4 firms with the 1 firm case corresponding to independent ordering. First, note that the contributions are ordered in the number of firms that participate in joint replenishment. Firms reduce their contributions as there are more firms in joint replenishment. As expected, the reduction is more significant when there are fewer firms. Second, as shown in Corollary 1, in equilibrium, a firm's contribution increases as its adjusted demand rate increases regardless of the number of firms participating in joint replenishment. One can also see that as the distribution shifts to the right (The most-likely value goes from 2 to 4 ), contributions for joint replenishments decrease, since the firm anticipates that other firms will have larger adjusted demand rates and contribute less. In some cases, a firm with a low adjusted demand rate may choose to contribute close to zero (but not zero), especially when there are more firms in joint replenishment (the bottom two plots when $m=3$ and $m=4$ ).

Fig. 3 shows the impact of asymmetric information on equilibrium under the same settings when there are two firms. The solid lines in Fig. 3 represent the expected contribution by a firm as a function of its own adjusted demand rate, given that it knows the adjusted demand rates of other firms in the joint replenishment program (full information). The dotted lines show the equilibrium contributions under asymmetric information. For lower values of adjusted demand rate, a firm that is not informed about its rivals' adjusted demand rates contributes more than what it would contribute on the average under full information. However, the full information contribution surpasses asymmetric information for higher levels of adjusted demand rate. As the distribution shifts to the right, contributions under both games decrease. However, contributions under full information are affected more, leading to a larger region over which asymmetric information contributions are larger than expected full information contributions.

Fig. 4 shows the impact of asymmetric information on equilibrium for three firms. Having three firms instead of two reduces contributions in both games. The shape of the contribution function for the asymmetric information game, however, does not change significantly. On the other hand, expected full information contributions are flatter when the adjusted demand rate is low. Overall, contributions decrease more in the asymmetric information game as the number of firms increase from two to three. This leads to a larger region over which full information contributions are larger. 

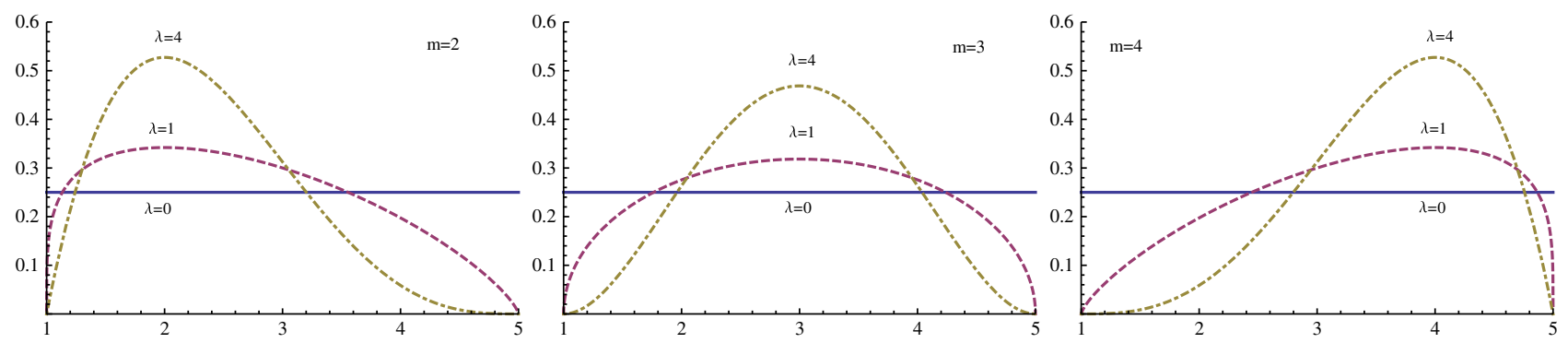

Fig. 1. The density function of modified PERT distribution $(a=1, b=5)$.
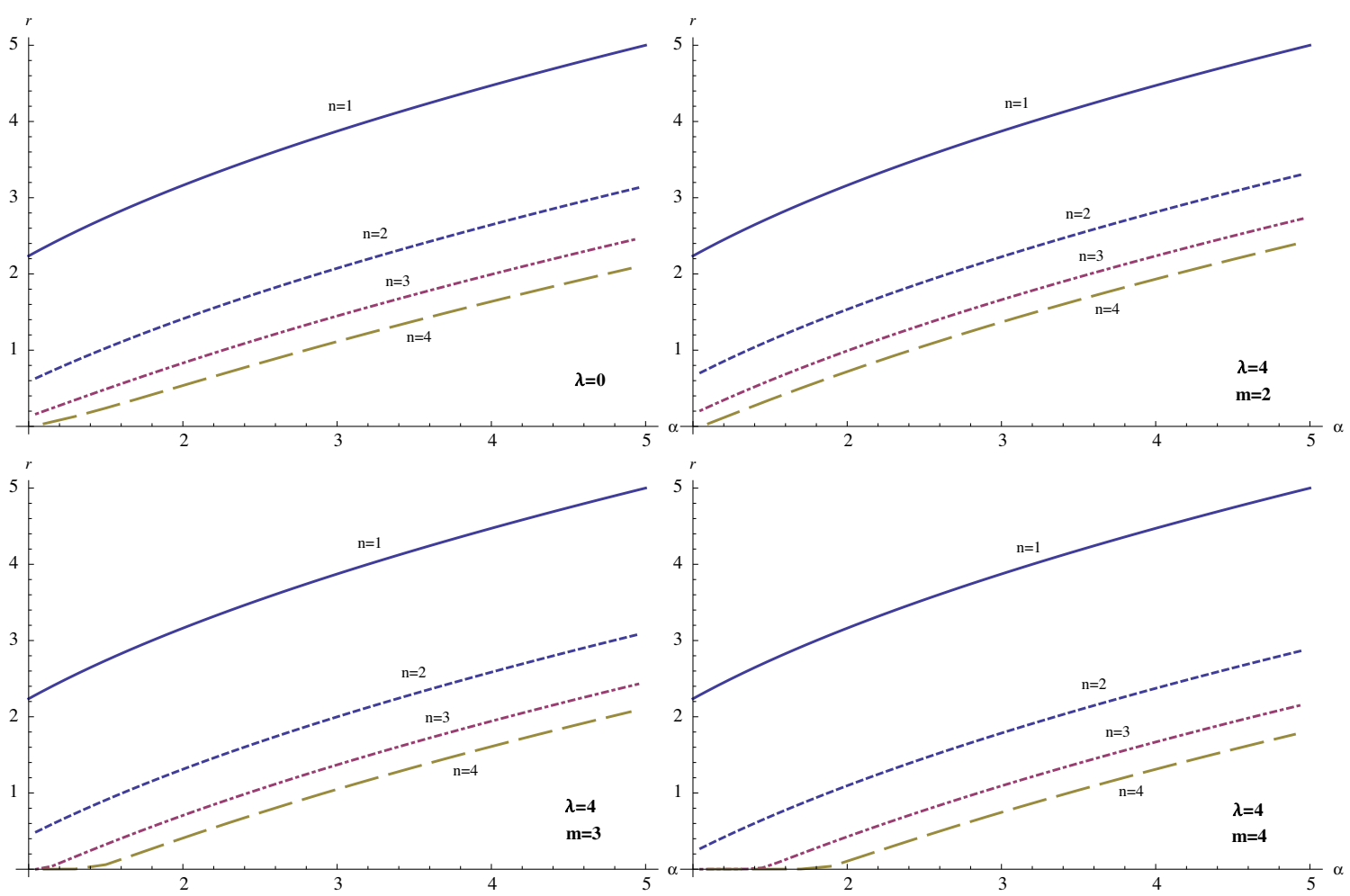

Fig. 2. Equilibrium contribution as a function of adjusted demand rate with $1,2,3$ and 4 firms under asymmetric information $(a=1, b=5)$

In order to better understand the effects of competition and information asymmetry on cycle times, aggregate contributions and aggregate total costs, we carried out a more detailed study in Table 1 . We use $a=1$ and consider two different values for $b$ : 5 and 9 . For $b=5$, the most-likely value $m$ takes on three values 2,3 and 4 . For $b=9$, we consider the values 3,5 , and 7 for $m$. The shape parameter $\lambda$ takes on three different values 0,1 , and 4 .

We consider cases with 2,3 , and 4 players. In order to provide a benchmark, we also show the results for cooperative joint replenishment and independent ordering. Since cooperative joint replenishment leads to lowest aggregate total costs, we use its expected aggregate total costs in (Column 8) as our baseline. Columns 9, 10, and 11 show the percentage deviation from the base case, of independent ordering, non-cooperative joint replenishment under asymmetric information and non-cooperative joint replenishment under full information, respectively. In these columns, we provide a policy's ex-ante performance against centralized ordering, i.e., if $C^{y}$ is the expected cost under policy $y$, we report $\Delta C^{y}=100 \times \frac{\mathbb{E}\left[C^{y}\right]-\mathbb{E}\left[C^{c}\right]}{\left[C^{c}\right]}$, where $C^{c}$ is expected cost under centralized ordering. In the last column of Table 1 , we report the percentage gap between aggregate total costs of full and asymmetric information games given by $\Delta C_{f}^{a}=100 \times\left(\mathbb{E}\left[C^{a}\right]-\mathbb{E}\left[C^{f}\right]\right) / \mathbb{E}\left[C^{f}\right]$.
We first note that the performance of independent ordering is not very sensitive to the distribution parameters for a given $n$. The aggregate total costs under independent ordering are, on the average, $40.22 \%, 71.26 \%$, and $97.49 \%$ higher than centralized ordering for $n=2,3$, and 4 , respectively. Note that this performance is very close to $100 \times(\sqrt{n}-1)$ which is equal to the percentage increase in aggregate total costs as one moves from centralized ordering to decentralized ordering when the firms are identical and their adjusted demands are deterministic (one can show this by observing that $C^{c}=\sqrt{2 n \kappa \alpha}$ and $C^{d}=n \sqrt{2 \kappa \alpha}$ if $\alpha_{j}$ is known and fixed at $\alpha$ for all $j \in N$ ).

Contributions under full information lead to significantly more efficient outcomes. The aggregate total costs, on the average, are only $3.61 \%, 9.40 \%$, and $15.53 \%$ higher than centralized ordering for $n=2,3$, and 4 , respectively. In the full information game, the efficiency is more sensitive to probability distribution of the adjusted demand rate. Note that the variability gets larger as $\lambda$ goes from 4 to 1 and then to 0 . The variability is also larger when the range goes from $(1,5)$ to $(1,9)$. In both of these cases, the efficiency of competitive full information game improves. Finally, as the distribution shifts to the left, the full information game also becomes more efficient. 

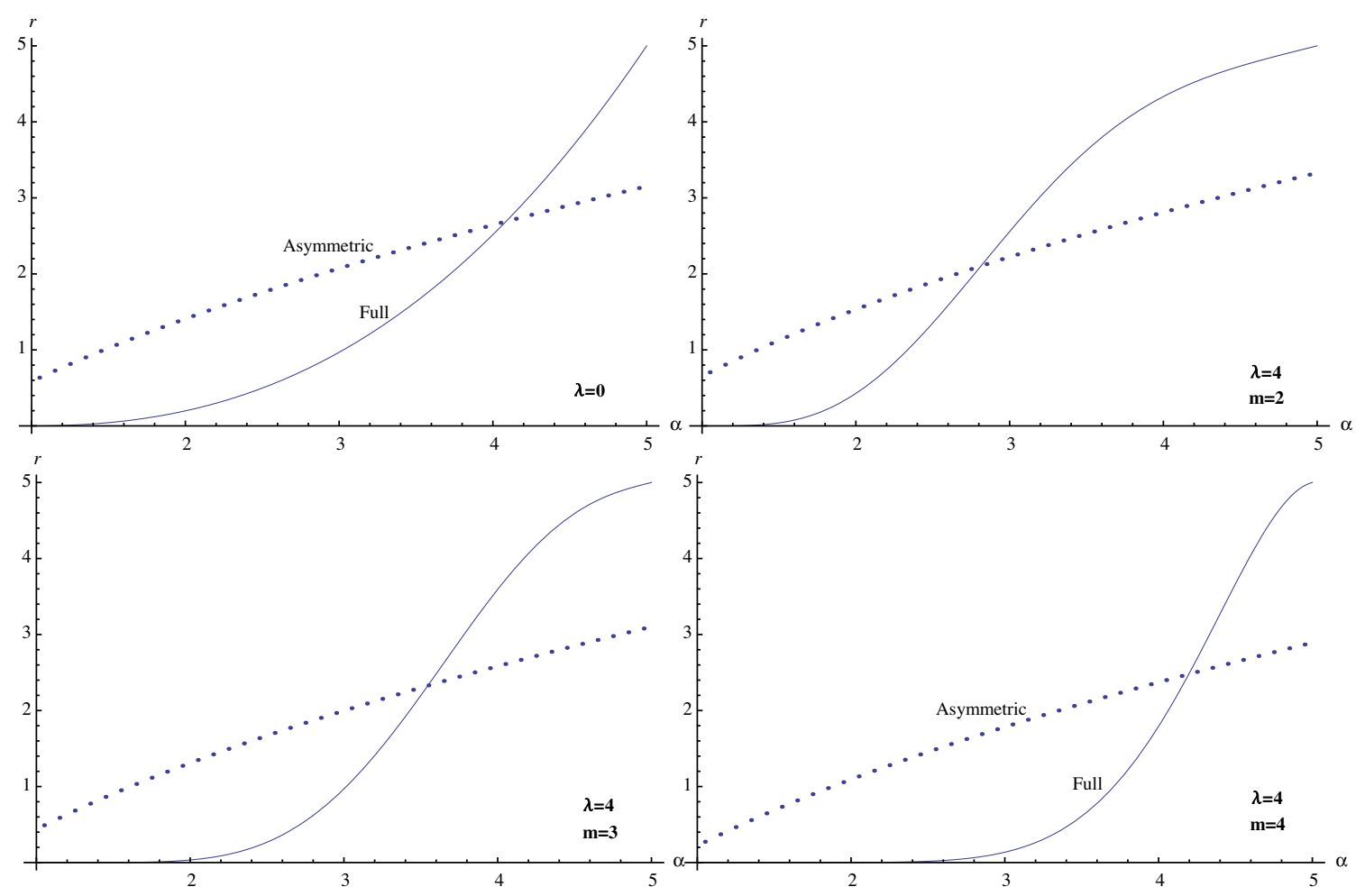

Fig. 3. Equilibrium (expected) contribution as a function of adjusted demand rate for two firms under asymmetric (full) information $(a=1, b=5)$.
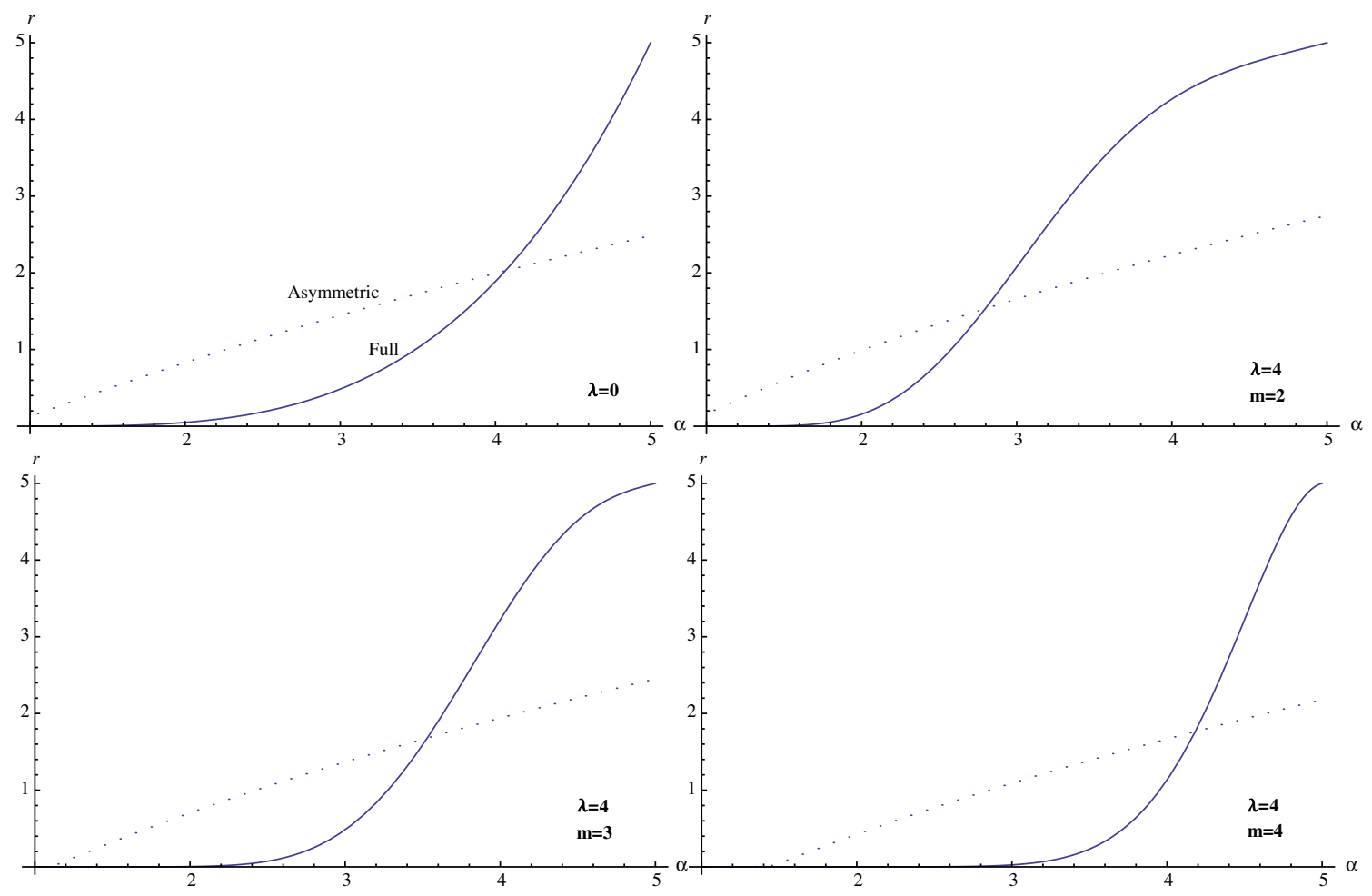

Fig. 4. Equilibrium (expected) contribution as a function of adjusted demand rate for three firms under asymmetric (full) information $(a=1, b=5)$.

While the asymmetric information game eliminates free-riding and ensures that firms contribute to joint replenishment, it also leads to firms with higher adjusted demand rates contribute less (see Figs. 3 and 4). The net effect is that aggregate contributions in the asymmetric information game are smaller than aggregate contributions in the full information game. Consequently, aggregate total costs are always larger in the asymmetric information game. The aggregate total costs, on the average, are $5.71 \%$, $14.05 \%$, and $22.18 \%$ higher than centralized ordering for $n=2,3$, and 4 , respectively. The performance of the asymmetric information game is quite insensitive to demand distribution. However, the effects of uncertainty and skewness can still be observed and are same as what we see in the full information game. As variability goes up and the distribution shifts to the left, the efficiency of 
Table 1

Performance comparisons.

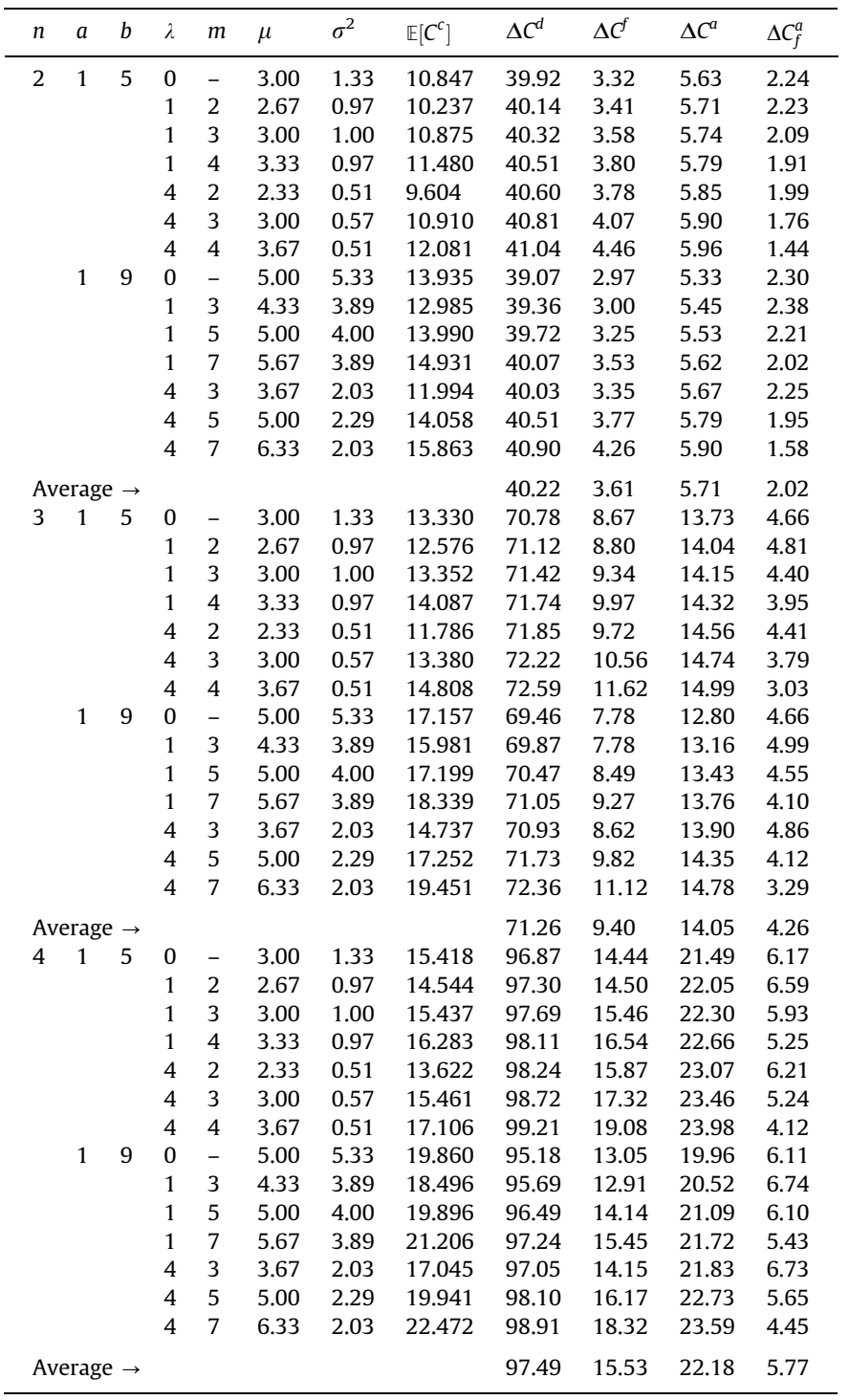

the asymmetric information game gets better. However, in comparison to the full information game, the gap is smaller when the variability is smaller and the distribution is right-skewed. Also the performance of asymmetric information compared to full information gets worse as the number of firms increase. This happens because total contributions decrease more for the asymmetric information game as more retailers participate in joint replenishment (See Figs. 3 and 4).

We should note that the gaps reported here between the centralized ordering and the asymmetric information game may be considered significant in practice. One may find it attractive to centralize the joint replenishment decision and allocate the efficient costs to the firms, especially given that stable allocations can be computed easily, at least in practice (Dror et al., 2012). However, any such cooperative solution requires information on all adjusted demand rates, which the firms may not want to disclose to the intermediary or each other for various reasons.

\section{Conclusion}

In this paper, we consider a joint replenishment problem in which $n$ ex-ante identical firms submits their contributions to an intermediary which sets a joint cycle time that would finance the fixed cost of replenishment. The demand rates and inventory holding cost rates are firms' private information. We show that an equilibrium for this asymmetric information game exists and characterize the equilibrium. We show that while information asymmetry eliminates free-riding in the contributions game, it also leads to contributing less for those firms who have higher demand rates and/or inventory holding cost rates. The net effect of information asymmetry is lower aggregate contributions and higher aggregate costs for the system.

One can consider a number of extensions to better capture the details of more realistic settings. First, one can consider more complex mechanisms that govern participation in joint replenishment. For example, one can investigate alternative mechanisms in which firms contribute sequentially rather than simultaneously or firms' contributions are stated as a function of joint cycle time. Second, one can better model the joint replenishment environment. For example, the replenishment cost (a fixed cost in our setting) may be a function of the volume or may involve minor costs. Finally, one may consider settings in which the a retailer may be facing uncertain and non-stationary demand.

\section{Appendix A}

\section{A.1. Proof of Lemma 1}

Consider the best response function for firm $j$ at $\alpha_{j}$ and $\alpha_{j}+\epsilon$ for a given $\epsilon>0$. First assume that $\alpha_{j}>\hat{\alpha}\left(\boldsymbol{\alpha}_{-\mathbf{j}}\right)$ and the best responses at $\alpha_{j}$ and $\alpha_{j}+\epsilon$ are interior solutions to problem in (4). In this case, firm $j$ 's best responses can be found by the following two equations:

$$
\begin{aligned}
& \int_{A^{n-1}} \frac{f^{n-1}\left(\boldsymbol{\alpha}_{-\mathbf{j}}\right)}{\left(\rho\left(\boldsymbol{r}_{-\mathbf{j}}, \alpha_{j}\right)+\sum_{k \in N, k \neq j} r_{k}\left(\alpha_{k}\right)\right)^{2}} \mathrm{~d} \boldsymbol{\alpha}_{-\mathbf{j}}=\frac{2}{\kappa \alpha_{j}}, \\
& \int_{A^{n-1}} \frac{f^{n-1}\left(\boldsymbol{\alpha}_{-\mathbf{j}}\right)}{\left(\rho\left(\boldsymbol{r}_{-\mathbf{j}}, \alpha_{j}+\epsilon\right)+\sum_{k \in N, k \neq j} r_{k}\left(\alpha_{k}\right)\right)^{2}} \mathrm{~d} \boldsymbol{\alpha}_{-\mathbf{j}}=\frac{2}{\kappa\left(\alpha_{j}+\epsilon\right)} .
\end{aligned}
$$

Taking the difference between (16) and (17) and rearranging, we get

$$
\begin{aligned}
& \int_{A^{n-1}} \frac{\left(\rho\left(\boldsymbol{r}_{-\mathbf{j}}, \alpha_{j}+\epsilon\right)+\rho\left(\boldsymbol{r}_{-\mathbf{j}}, \alpha_{j}\right)+2 \sum_{k \in N, k \neq j} r_{k}\left(\alpha_{k}\right)\right) f^{n-1}\left(\boldsymbol{\alpha}_{-\mathbf{j}}\right)}{\left(\rho\left(\boldsymbol{r}_{-\mathbf{j}}, \alpha_{j}+\epsilon\right)+\sum_{k \in N, k \neq j} r_{k}\left(\alpha_{k}\right)\right)^{2}\left(\rho\left(\boldsymbol{r}_{-\mathbf{j}}, \alpha_{j}\right)+\sum_{k \in N, k \neq j} r_{k}\left(\alpha_{k}\right)\right)^{2}} \mathrm{~d} \boldsymbol{\alpha}_{-\mathbf{j}} \\
& =\frac{2 \epsilon}{\kappa \alpha_{j}\left(\alpha_{j}+\epsilon\right)\left(\rho\left(\boldsymbol{r}_{-\mathbf{j}}, \alpha_{j}+\epsilon\right)-\rho\left(\boldsymbol{r}_{-\mathbf{j}}, \alpha_{j}\right)\right)} .
\end{aligned}
$$

The integrand in the left hand side of (18) is strictly positive. Therefore, the definite integral should also be positive, leading to $\rho\left(\boldsymbol{r}_{-\mathbf{j}}, \alpha_{j}+\epsilon\right)>\rho\left(\boldsymbol{r}_{-\mathbf{j}}, \alpha_{j}\right)$.

On the other hand, if $\alpha_{j} \leqslant \hat{\alpha}\left(\boldsymbol{\alpha}_{-\mathbf{j}}\right)$ and firm $j$ 's best response is to contribute zero at $\alpha_{j}$, then the firm will contribute either zero or a positive amount at $\alpha_{j}+\epsilon$.

\section{A.2. Proof of Theorem 1}

In order to prove the existence we invoke the following proposition by Meirowitz (2003):

Proposition 1. A Bayesian game has a pure-strategy Bayesian Nash equilibrium if for each $j \in N$

1. A and $\Theta$ are nonempty, convex and compact subsets of Euclidean space.

2. $u_{j}(r, \alpha)=-\phi_{j}(r, \alpha)$ is continuous.

3. For every $\alpha$ and measurable function $f^{n-1}\left(\alpha_{-\mathbf{j}}\right)$ 


$$
\int_{A^{n-1}} u_{j}\left(r_{j}, \boldsymbol{r}_{-\mathbf{j}}, \alpha_{j}, \boldsymbol{\alpha}_{-\mathbf{j}}\right) f^{n-1}\left(\boldsymbol{\alpha}_{-\mathbf{j}}\right) \mathrm{d} \boldsymbol{\alpha}_{-\mathbf{j}}=-\Phi_{j}\left(r_{j}, \boldsymbol{r}_{-\mathbf{j}}, \alpha_{j}\right)
$$

is strictly quasi-concave in $r_{j}$.

4. For every $\varepsilon_{j}>0$ there exists some constant $v_{j}$ such that for any given $r_{-\mathbf{j}}$ then

$$
\sup _{\left\{\left(\alpha_{j}, \alpha_{j}^{\prime}\right) \in A:\left|\alpha_{j}-\alpha_{j}^{\prime}\right|<v_{j}\right.}\left|\rho\left(\boldsymbol{r}_{-\mathbf{j}}, \alpha_{j}\right)-\rho\left(\boldsymbol{r}_{-\mathbf{j}}, \alpha_{j}^{\prime}\right)\right|<\varepsilon_{j} .
$$

5. $f^{n-1}\left(\alpha_{-\mathbf{j}}\right)$ is continuous.

Now, $A$ and $\Theta$ are both closed, bounded and consist of single intervals by assumption. Thus, they are nonempty, convex and compact. Thus, condition 1 is satisfied. Since the function $\phi$ given in Eq. (2) is continuous, condition 2 is also satisfied. Similarly, the belief function $f^{n-1}\left(\boldsymbol{\alpha}_{-\mathbf{j}}\right)$ is continuous since it is the multiplication of continuous probability density functions $f(\alpha)$ by assumption so condition 5 is satisfied. Since the derivative of $\Phi$ provided in (6) is strictly positive, $\Phi$ is convex. Therefore condition 3 is also satisfied.

The only remaining condition is condition 4 , which states that the slope of the best response function is uniformly bounded. In order to prove the assertion, we take two different types $\alpha_{j}$ and $\alpha_{j}^{\prime}$ for firm $j$. First, assume that the best responses at $\alpha_{j}$ and $\alpha_{j}^{\prime}$ both satisfy the first order conditions (i.e., they are different from zero).

$$
\begin{aligned}
\frac{2}{\kappa \alpha_{j}}-\frac{2}{\kappa \alpha_{j}^{\prime}}= & \int_{A^{n-1}} \frac{1}{\left(\rho\left(\boldsymbol{r}_{-\mathbf{j}}, \alpha_{j}\right)+\sum_{i \neq j} r_{i}\left(\alpha_{i}\right)\right)^{2}} f^{n-1}\left(\boldsymbol{\alpha}_{-\mathbf{j}}\right) \mathrm{d} \boldsymbol{\alpha}_{-\mathbf{j}} \\
& -\int_{A^{n-1}} \frac{1}{\left(\rho\left(\boldsymbol{r}_{-\mathbf{j}}, \alpha_{j}^{\prime}\right)+\sum_{i \neq j} r_{i}\left(\alpha_{i}\right)\right)^{2}} f^{n-1}\left(\boldsymbol{\alpha}_{-\mathbf{j}}\right) \mathrm{d} \boldsymbol{\alpha}_{-\mathbf{j}} \\
& =\int_{A^{n-1}}\left(\frac{1}{\left(\rho\left(\boldsymbol{r}_{-\mathbf{j}}, \alpha_{j}\right)+\sum_{i \neq j} r_{i}\left(\alpha_{i}\right)\right)^{2}}-\frac{1}{\left(\rho\left(\boldsymbol{r}_{-\mathbf{j}}, \alpha_{j}^{\prime}\right)+\sum_{i \neq j} r_{i}\left(\alpha_{i}\right)\right)^{2}} f^{n-1}\left(\boldsymbol{\alpha}_{-\mathbf{j}}\right)\right) \mathrm{d} \boldsymbol{\alpha}_{-\mathbf{j}} \\
& =\int_{A^{n-1}} \frac{\left(\rho\left(\boldsymbol{r}_{-\mathbf{j}}, \alpha_{j}^{\prime}\right)+\sum_{i \neq j} r_{i}\left(\boldsymbol{\alpha}_{i}\right)\right)^{2}-\left(\rho\left(\boldsymbol{r}_{-\mathbf{j}}, \alpha_{j}\right)+\sum_{i \neq j} r_{i}\left(\alpha_{i}\right)\right)^{2}}{\left(\rho\left(\boldsymbol{r}_{-\mathbf{j}}, \alpha_{j}\right)+\sum_{i \neq j} r_{i}\left(\alpha_{i}\right)\right)^{2}\left(\rho\left(\boldsymbol{r}_{-\mathbf{j}}, \alpha_{j}^{\prime}\right)+\sum_{i \neq j} r_{i}\left(\alpha_{i}\right)\right)^{2}} f^{n-1}\left(\boldsymbol{\alpha}_{-\mathbf{j}}\right) \mathrm{d} \boldsymbol{\alpha}_{-\mathbf{j}} \\
& =\left(\rho\left(\boldsymbol{r}_{-\mathbf{j}}, \alpha_{j}^{\prime}\right)-\rho\left(\boldsymbol{r}_{-\mathbf{j}}, \alpha_{j}\right)\right) \\
& \times \int_{A^{n-1}} \frac{\rho\left(\boldsymbol{r}_{-\mathbf{j}}, \alpha_{j}\right)+\rho\left(\boldsymbol{r}_{-\mathbf{j}}, \alpha_{j}^{\prime}\right)+2 \sum_{i \neq j} r_{i}\left(\alpha_{i}\right)}{\left(\rho\left(\boldsymbol{r}_{-\mathbf{j}}, \boldsymbol{\alpha}_{j}\right)+\sum_{i \neq j} r_{i}\left(\alpha_{i}\right)\right)^{2}\left(\rho\left(\boldsymbol{r}_{-\mathbf{j}}, \alpha_{j}^{\prime}\right)+\sum_{i \neq j} r_{i}\left(\alpha_{i}\right)\right)^{2}} f^{n-1}\left(\boldsymbol{\alpha}_{-\mathbf{j}}\right) \mathrm{d} \boldsymbol{\alpha}_{-\mathbf{j}} \\
& =\left(\rho\left(\boldsymbol{r}_{-\mathbf{j}}, \alpha_{j}^{\prime}\right)-\rho\left(\boldsymbol{r}_{-\mathbf{j}}, \alpha_{j}\right)\right) \\
& \times\left(\int_{A^{n-1}} \frac{1}{\left(\rho\left(\boldsymbol{r}_{-\mathbf{j}}, \alpha_{j}\right)+\sum_{i \neq j} r_{i}\left(\alpha_{i}\right)\right)\left(\rho\left(\boldsymbol{r}_{-\mathbf{j}}, \alpha_{j}^{\prime}\right)+\sum_{i \neq j} r_{i}\left(\alpha_{i}\right)\right)^{2}} f^{n-1}\left(\boldsymbol{\alpha}_{-\mathbf{j}}\right) \boldsymbol{\alpha}_{-\mathbf{j}}\right. \\
& \left.+\int_{A^{n-1}} \frac{1}{\left(\rho\left(\boldsymbol{r}_{-\mathbf{j}}, \alpha_{j}\right)+\sum_{i \neq j} r_{i}\left(\alpha_{i}\right)\right)^{2}\left(\rho\left(\boldsymbol{r}_{-\mathbf{j}}, \alpha_{j}^{\prime}\right)+\sum_{i \neq j} r_{i}\left(\alpha_{i}\right)\right)} f^{n-1}\left(\boldsymbol{\alpha}_{-\mathbf{j}}\right) \mathrm{d} \boldsymbol{\alpha}_{-\mathbf{j}}\right)
\end{aligned}
$$

Using the first order conditions and $r_{j}\left(\alpha_{j}\right) \leqslant \bar{r}$ for all $j \in N$, we can write

$$
\begin{gathered}
\int_{A^{n-1}} \frac{1}{\left(\rho\left(\boldsymbol{r}_{-\mathbf{j}}, \alpha_{j}\right)+\sum_{i \neq j} r_{i}\left(\alpha_{i}\right)\right)^{2}\left(\rho\left(\boldsymbol{r}_{-\mathbf{j}}, \alpha_{j}\right)+\sum_{i \neq j} r_{i}\left(\alpha_{i}\right)\right)} f^{n-1}\left(\boldsymbol{\alpha}_{-\mathbf{j}}\right) \mathrm{d} \boldsymbol{\alpha}_{-\mathbf{j}} \\
\geqslant \int_{A^{n-1}} \frac{1}{n \bar{r}\left(\rho\left(\boldsymbol{r}_{-\mathbf{j}}, \alpha_{j}\right)+\sum_{i \neq j} r_{i}\left(\alpha_{i}\right)\right)^{2}} f^{n-1}\left(\boldsymbol{\alpha}_{-\mathbf{j}}\right) \boldsymbol{\alpha}_{-\mathbf{j}}=\frac{2}{n \bar{r} \kappa \alpha_{j}} .
\end{gathered}
$$

A similar result can be obtained for $\alpha_{j}^{\prime}$. Taking the absolute values on both sides, we have

$$
\left|\frac{2}{\kappa \alpha_{j}}-\frac{2}{\kappa \alpha_{j}^{\prime}}\right| \geqslant\left|\rho\left(\boldsymbol{r}_{-\mathbf{j}}, \alpha_{j}^{\prime}\right)-\rho\left(\boldsymbol{r}_{-\mathbf{j}}, \alpha_{j}\right)\right|\left(\frac{2}{n \bar{r} \kappa \alpha_{j}}+\frac{2}{n \bar{r} \kappa \alpha_{j}^{\prime}}\right) .
$$

Rearranging the terms and using $\alpha_{j} \geqslant \underline{\alpha}>0$ and $\alpha_{j}^{\prime} \geqslant \underline{\alpha}>0$, we obtain:

$$
\begin{aligned}
\left|\alpha_{j}^{\prime}-\alpha_{j}\right| & \geqslant\left|\rho\left(\boldsymbol{r}_{-\mathbf{j}}, \alpha_{j}^{\prime}\right)-\rho\left(\boldsymbol{r}_{-\mathbf{j}}, \alpha_{j}\right)\right|\left(\frac{\alpha_{j}^{\prime}+\alpha_{j}}{n \bar{r}}\right) \\
& \geqslant\left|r_{j}^{*}\left(\alpha_{j}^{\prime}\right)-r_{j}^{*}\left(\alpha_{j}\right)\right|(2 \underline{\alpha} / n \bar{r}) .
\end{aligned}
$$

Now, assume that $\varepsilon_{j}>0$ and let $v_{j}=\varepsilon(2 \underline{\alpha} / n \bar{r})$. Then $\left|\alpha_{j}^{\prime}-\alpha_{j}\right|<v_{j}$ implies $\left|\rho\left(\boldsymbol{r}_{-\mathbf{j}}, \alpha_{j}^{\prime}\right)-\rho\left(\boldsymbol{r}_{-\mathbf{j}}, \alpha_{j}\right)\right|(2 \underline{\alpha} / n \bar{r})<v_{j}$ or $\left|r_{j}^{*}\left(\alpha_{j}^{\prime}\right)-r_{j}^{*}\left(\alpha_{j}\right)\right|<\varepsilon_{j}$.

The fact that the best response function has a uniformly bounded slope can also be shown more easily if we have $\rho\left(\boldsymbol{r}_{-\mathbf{j}}\right.$, $\left.\alpha_{j}\right)=0$ and/or $\rho\left(\boldsymbol{r}_{-\mathbf{j}}, \alpha_{j}^{\prime}\right)=0$.

\section{A.3. Proof of Lemma 2}

Assume that for all $k \neq j$, there exists $B_{k} \subset A$ such that $B_{k} \neq \emptyset$ and $r_{k}^{*}\left(\alpha_{k}\right)=0$ for $\alpha_{k} \in B_{k}$. Let $B=\prod_{k \neq j} B_{k}$. Given its type and other firms' equilibrium contributions, firm $j$ 's best response is to select a contribution $r_{j} \geqslant 0$ that minimizes

$$
\begin{aligned}
\Phi_{j}\left(r_{j}, \boldsymbol{r}_{-\mathbf{j}}^{*}, \alpha_{j}\right)= & \frac{1}{2} \kappa \alpha_{j} \int_{B} \frac{1}{r_{j}} f^{n-1}\left(\boldsymbol{\alpha}_{-\mathbf{j}}\right) \mathrm{d} \boldsymbol{\alpha}_{-\mathbf{j}}+\frac{1}{2} \kappa \alpha_{j} \int_{A^{n-1} \backslash B} \\
& \times \frac{1}{r_{j}+\sum_{k \in N, k \neq j} r_{k}^{*}\left(\alpha_{k}\right)} f^{n-1}\left(\boldsymbol{\alpha}_{-\mathbf{j}}\right) \mathrm{d} \boldsymbol{\alpha}_{-\mathbf{j}}+r_{j} .
\end{aligned}
$$

Clearly, firm $j$ 's best response is to select $r_{j}$ that is strictly positive for any given $\alpha_{j}$. This leads to an equilibrium contribution function that satisfies $r_{j}^{*}\left(\alpha_{j}\right)>0$ for all $\alpha_{j}$.

\section{A.4. Proof of Theorem 2}

Let $j$ be the firm which satisfies $r^{*}\left(\alpha_{j}\right)>0$ for all $\alpha_{j} \in A$. Note that $r_{j}^{*}\left(\alpha_{j}\right)$ is firm $j$ 's best response to other firms' equilibrium contributions. Since $r_{j}^{*}\left(\alpha_{j}\right)>0$ for all $\alpha_{j}$, we have the following from (7)

$$
\begin{aligned}
& \int_{A^{n-1}} \frac{1}{\left(r_{1}^{*}\left(\alpha_{1}\right)+r_{2}^{*}\left(\alpha_{2}\right)+\cdots+r_{n}^{*}\left(\alpha_{n}\right)\right)^{2}} f^{n-1}\left(\boldsymbol{\alpha}_{-\mathbf{j}}\right) \mathrm{d} \boldsymbol{\alpha}_{-\mathbf{j}} \\
& \quad=\frac{2}{\kappa \alpha_{j}} \text { for all } \alpha_{j} .
\end{aligned}
$$

Multiplying both sides of Eq. (19) by $f\left(\alpha_{j}\right)$ and integrating over $A$ we get

$\int_{A^{n}} \frac{1}{\left(r_{1}^{*}\left(\alpha_{1}\right)+r_{2}^{*}\left(\alpha_{2}\right)+\cdots+r_{n}^{*}\left(\alpha_{n}\right)\right)^{2}} f^{n}(\boldsymbol{\alpha}) \mathrm{d} \boldsymbol{\alpha}=\mathbb{E}\left[\frac{2}{\kappa \alpha_{j}}\right]$

Now consider another firm $k$ such that

$$
\int_{A^{n-1}} \frac{1}{\left(r_{1}^{*}\left(\alpha_{1}\right)+r_{2}^{*}\left(\alpha_{2}\right)+\cdots+r_{n}^{*}\left(\alpha_{n}\right)\right)^{2}} f^{n-1}\left(\boldsymbol{\alpha}_{-\mathbf{k}}\right) \mathrm{d} \boldsymbol{\alpha}_{-\mathbf{k}}+\zeta_{k}\left(\alpha_{k}\right)=\frac{2}{\kappa \alpha_{k}}
$$

where $\zeta_{k}\left(\alpha_{k}\right)$ is zero when $r_{k}^{*}\left(\alpha_{k}\right)>0$ and $\zeta_{k}\left(\alpha_{k}\right)$ is possibly a positive quantity when $r_{k}^{*}\left(\alpha_{k}\right)=0$. Multiplying each term of Eq. (21) by $f\left(\alpha_{k}\right)$ and integrating over $A$, we get

$$
\begin{aligned}
& \int_{A^{n}} \frac{1}{\left(r_{1}^{*}\left(\alpha_{1}\right)+r_{2}^{*}\left(\alpha_{2}\right)+\cdots+r_{n}^{*}\left(\alpha_{n}\right)\right)^{2}} f^{n}(\boldsymbol{\alpha}) \mathrm{d} \boldsymbol{\alpha}+\mathbb{E}\left[\zeta_{k}\left(\alpha_{k}\right)\right] \\
& \quad=\mathbb{E}\left[\frac{2}{\kappa \alpha_{k}}\right]
\end{aligned}
$$

Using (20) and (22) we get

$\mathbb{E}\left[\zeta_{k}\left(\alpha_{k}\right)\right]=\mathbb{E}\left[\frac{2}{\kappa \alpha_{k}}\right]-\mathbb{E}\left[\frac{2}{\kappa \alpha_{j}}\right]$.

Since $\alpha_{j}$ and $\alpha_{k}$ are identically distributed, the right hand side of (23) is zero leading to $\mathbb{E}\left[\zeta_{k}\left(\alpha_{k}\right)\right]=0$. Note however that $\zeta_{k}\left(\alpha_{k}\right)$ can only take on non-negative values. Therefore, we should have $\zeta_{k}\left(\alpha_{k}\right)=0$ for all $\alpha_{k}$ leading to Eq. (19) being valid not only for firm $j$ but for all other firms. 


\section{References}

Anily, S., Haviv, M., 2007. The cost allocation problem for the first order interaction joint replenishment model. Operations Research 55, 292-302.

Bauso, D., Giarre, L., Pesenti, R., 2008. Consensus in noncooperative dynamic games: a multiretailer inventory application. IEEE Transactions on Automatic Contro 53, 998-1003.

Burnetas, A., Gilbert, S.M., Smith, C.E., 2007. Quantity discounts in single-period supply contracts with asymmetric demand information. IIE Transactions 39, 465-479.

Clemons, E., Reddi, S., Row, M., 1993. The impact of information technology on the organization of economic activity: the "move to the middle" hypothesis. Journal of Management Information Systems 10, 9-35.

Corbett, C.J., 2001. Stochastic inventory systems in a supply chain with asymmetric information: cycle stocks, safety stocks, and consignment stock. Operations Research 49, 487-500.

Dror, M., Hartman, B., Chang, W., 2012. The cost allocation issue in join replenishment. International Journal of Production Economics 135, 242-254.

Elomri, A., Ghaffari, A., Jemai, Z., Dallery, Y., 2012. Coalition formation and cost allocation for joint replenishment systems. Production and Operations Management 21, 1015-1027.

Fiestras-Janeiro, M.G., Meca, A., Mosquera, M.A., 2011. Cooperative game theory and inventory management. European Journal of Operational Research 210, 459-466.

Harris, F.W., 1913. How many parts to make at once. Factory, The Magazine of Management 10, 135-136, 152.

Hartman, B., Dror, M., 2007. Shipment consolidation: who pays for it and how much? Management Science 53, 78-87.
Jans, R., Degraeve, Z., 2008. Modeling industrial lot sizing problems: a review. International Journal of Production Research 46, 1619-1643.

Khouja, M., Goyal, S., 2008. A review of the joint replenishment problem literature: 1989-2005. European Journal of Operational Research 86, 1-16.

Körpeoğlu, E., Sen, A., Güler, K., 2012. A private contributions game for joint replenishment. Mathematical Methods of Operations Research 75, 67-82.

Meca, A., Garcia-Jurado, I., Borm, P., 2003. Cooperation and competition in inventory games. Mathematical Methods of Operations Research 57, 481-493.

Meca, A., Timmer, J., Garcia-Jurado, I., Borm, P., 2004. Inventory games. European Journal of Operational Research 156, 127-139.

Meirowitz, A., 2003. On the existence of equilibria to Bayesian games with nonfinite type and action spaces. Economic Letters 78, 213-218.

Minner, S., 2007. Bargaining for cooperative economic ordering. Decision Support Systems 43, 569-583.

Nagarajan, M., Sošić, G., 2008. Game theoretic analysis of cooperation among supply chain agents: Review and extensions. European Journal of Operational Research 187, 719-745

Van den Heuvel, W., Borm, P., Hamers, H., 2007. Economic lot-sizing games. European Journal of Operational Research 176, 1117-1130.

Viswanathan, S., Piplani, R., 2001. Coordinating supply chain inventories through common replenishment epochs. European Journal of Operational Research 129, 277-286.

Vose, D., 2008. Risk Analysis: A Quantitative Guide, third ed. John Wiley \& Sons, West Sussex, England.

Zhang, J., 2009. Cost allocation for joint replenishment models. Operations Research 57, 146-156. 